\title{
IMIA Member Societies and Corresponding Members
}

\section{Member Societies}

AMIA (American Medical Informatics Association)

Argentine Association of Medical Informatics (AAIM)

Association for Health Informatics of Nigeria (AHIN)

Association for Medical and Bio-Informatics, Singapore (AMBIS)

Association for Medical Informatics of Serbia

Belgian Medical Informatics Association

Brazilian Society of Health Informatics (SBIS)

British Computer Society (BCS Health)

Burundi Health Informatics Association

Cameroonian Health Informatics Society (CAHIS)

Chilean Health Informatics Society

China Medical Informatics Association

COACH: Canada's Health Informatics Association

Colombian Association for Health Informatics

Croatian Society for Medical Informatics

Cuban Society of Medical Informatics

Czech Society for Biomedical Engineering and Medical Informatics

eHealth Association of Pakistan (eHAP)

Finnish Social and Health Informatics Association (FinnSHIA)

French Medical Informatics Association (AIM)

German Association for Medical Informatics, Biometry and Epidemiology (GMDS)

Ghana Health Informatics Association

Greek Health Informatics Association

Health Informatics New Zealand

Health Informatics Society of Australia Ltd. (HISA)

Health Informatics Society of Sri Lanka

Healthcare Informatics Society of Ireland

Hong Kong Society of Medical Informatics

Indian Association for Medical Informatics (IAMI)

Iranian Medical Informatics Association

Ivorian Society of Biosciences and Health Informatics (ISBHI)

\section{Corresponding Members}

Albania, Algeria, Armenia, Azerbaijan, Bangladesh, Democratic Republic of Congo, Egypt, El Salvador, Indonesia, Iraq, Jamaica, Jordan, Kuwait, Lebanon, Madagascar, Malaysia, Moldova, Nepal, Oman, Qatar, Russian Federation, Sudan, Syria, Tanzania, Trinidad \& Tobago, Uganda, United Arab Emirates, Uzbekistan, Zambia, Zimbabwe
Japan Association for Medical Informatics

John von Neumann Computer Society (Hungary)

Kenya Health Informatics Association

Korea Society of Medical Informatics (KOSMI)

Medical Informatics Association of Malawi (MIAM)

Mexican Medical Informatics Association

Norwegian Society for Medical Informatics

Peruvian Association of Biomedical Informatics

Philippine Medical Informatics Society

Romanian Society of Medical Informatics

Slovenian Medical Informatics Association (SIMIA)

Society for Medical Informatics of Bosnia and Herzegovina

South African Health Informatics Association

Spanish Society of Health Informatics

Swedish Federation for Medical Informatics (SFMI)

Swiss Society for Medical Informatics

Taiwan Association for Medical Informatics (TAMI)

Thai Medical Informatics Association

The Bolivian Medical Informatics and Telemedicine Society (SOBOTIM)

The Israeli Association for Medical Informatics (ILAMI)

The Mali Society of Biomedical and Health Information (SOMBIS)

The Saudi Association for Health Informatics (SAHI)

The Ukrainian Association for Computer Medicine (UACM)

Togolese Association for Medical Informatics and Telemedicine (ATIM-TELEMED)

Turkish Medical Informatics Association (TURKMIA)

Uruguayan Society of Health Informatics

Venezuelan Association of Computer Science in Health (AVIS)

VMBI, Society for Healthcare Informatics (The Netherlands)

Working Group Medical Informatics and eHealth of the Austrian Computer Society (OCG) and the Austrian Society for Biomedical Engineering (ÖGBMT)

\section{Affiliate Members}

International Federation for Information Processing (IFIP)

- www.ifip.org

International Federation of Health Information Management Associations (IFHIMA) - www.ifhima.org

World Health Organization - www.who.int 